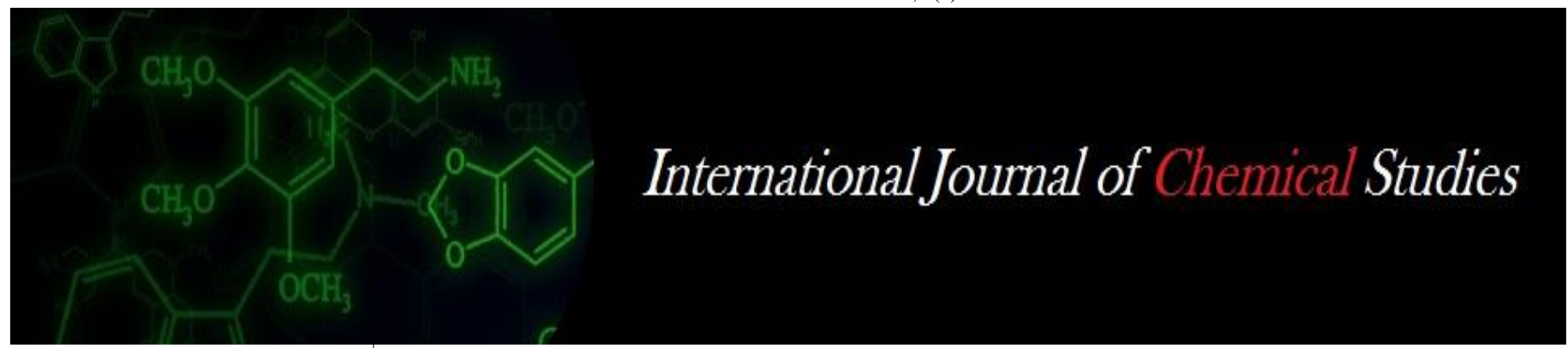

P-ISSN: 2349-8528

E-ISSN: 2321-4902

IJCS 2020; 8(1): 2306-2313

(C) 2020 IJCS

Received: 10-11-2019

Accepted: 13-12-2019

Gajendra Chandrakar

Department of Entomology,

Indira Gandhi Krishi

Vishwavidyalaya, Raipur,

Chhattisgarh, India

Renuka

Department of Entomology, Indira Gandhi Krishi

Vishwavidyalaya, Raipur,

Chhattisgarh, India

Manmohan Singh Bisen

Department of Entomology,

Indira Gandhi Krishi

Vishwavidyalaya, Raipur,

Chhattisgarh, India

Harshal Kumar Chandrakar Department of Entomology, Indira Gandhi Krishi

Vishwavidyalaya, Raipur,

Chhattisgarh, India
Corresponding Author: Renuka

Department of Entomology, Indira Gandhi Krishi

Vishwavidyalaya, Raipur,

Chhattisgarh, India

\section{Bio-efficacy of Emamectin Benzoate 3.5\% + lambda cyhalothrin 5\% WP against sucking insect pests of chilli during Rabi season 2017-18 and 2018-19}

\section{Gajendra Chandrakar, Renuka, Manmohan Singh Bisen and Harshal Kumar Chandrakar}

DOI: https://doi.org/10.22271/chemi.2020.v8.i1ai.8612

\begin{abstract}
The field experiment was conducted to evaluate the "Effect of soil and foliar supplementation of nitrogen, boron and salicylic acid on growth and yield of cucumber (Cucumis sativus L.) in alfisols of Konkan (M.S.)" at Research and Education Farm, Department of Agricultural Botany, College of Agriculture, Dr. Balasaheb Sawant Konkan Krishi Vidyapeeth, Dapoli, Dist. Ratnagiri (M.S.) during the Summer season of 2018. The experiment was laid out in Randomized Block Design (RBD) comprising ten treatment combinations replicated thrice, where the effect of soil and foliar supplementation of nitrogen, boron and salicylic acid either alone or in combinations applied along with the recommended dose of fertilizers (135:60:30 NPK kg ha-1) and an absolute control (to judge the fate of native nutrients) were studied. The study further revealed that the treatment receiving the application of recommended dose of fertilizer (135:60:30 $\left.\mathrm{kg} \mathrm{ha}^{-1}\right)+$ Foliar spray of nitrogen through urea (1\%) + Soil application of boron through borax @ $2 \mathrm{~kg} \mathrm{ha}^{-1}+$ Foliar spray of salicylic acid $(0.2 \%)$ was recorded the highest fruit yield $\left(231.22 \mathrm{q} \mathrm{ha}^{-1}\right)$ and yield attributing characters like number of fruits per vine (7.00) and weight of fruit i.e., $1.98 \mathrm{~kg}$ per vine as well as growth parameters viz.vine length $(407.00 \mathrm{~cm})$ and number of branches per vine (14.22) of Cucumber in alfisols of Konkan (M.S.).
\end{abstract}

Keywords: Emamectin Benzoate, salicylic acid, alfisols

\section{Introduction}

Chilli (Capsicum annum L.) popularly known as 'mirch' in Hindi. It belongs to the family Solanaceae. Chilli is one of the important vegetable and commercial spice crops (Mondal et al., 2012) ${ }^{[13]}$. The red color of chillies is due to the presence of pigment 'Capsanthin' (Choudhary et al., 2009) ${ }^{[3]}$ It is grown throughout the year as a cash crop and used in green and red ripe dried stage for their pungency, colour and other ingredients in all culinary preparations of rich and poor alike to impart taste, flavour and colour. Nutritionally, it is a rich source of vitamin A, B and C. Capsaicin an alkaloid responsible for pungency in chillies has medicinal properties and it prevents heart attack by dilating the blood vessels (Gill, 1989) ${ }^{[6]}$. Important chilli producing countrys are China, India, Indonesia and Sri Lanka in Asian region. Nigeria, Tunisia, Ghana, and Egypt in Africa, Mexico, United States of America in North \& Central America, Spain, Yugoslavia, Romania, Italy, Bulgaria, and Hungary in Europe and Peru, Argentina and Brazil in South America (Kraft et al., 2014) ${ }^{[10]}$. In India, chilli is grown in $2,87,050$ ha area with a production of 34,06,030 metric tonnes (Anonymous, 2017) ${ }^{[1]}$. Whereas in Chhattisgarh, the area is 37,320 ha and production is 2, 68, 440 metric tonnes (Anonymous, 2017) ${ }^{[1]}$. Indian Chilli is principally exported to Bahrain, Israel, Bangladesh, Japan, USA, Malaysia and UAE (Halder and Kodandaram, 2014) ${ }^{[7]}$.

The chilli crop is attacked by a number of insect-pests right from germination to harvest of the crop, out of them thrips is major sucking insect pests, responsible for low productivity, reduce up to 50 per cent yield (Ahmed et al., 1987) ${ }^{[2]}$. Under severe infestation thrips alone cause 50 percent yield loss (Kandasamy et al., 1990). The yield losses range from 50-90 percent due to insect pests of chilli (Nelson and Natrajan, 1994 and Kumar, 1995) ${ }^{[15,11]}$. These sucking pests causes serious damage to chilli crop by direct feeding and transmit deadly chilli leaf 
curl disease. Both adults and nymphs of thrips feed by rasping and sucking the oozing cell sap from the ventral side of the leaves, growing shoots, developing flowers and fruits. The affected leaves curl and exhibit characteristic leaf curl symptoms. Over use of pesticides has often led to the development of undesirable problems like destruction of natural enemies, pest resurgence and failure of control strategies leading to outbreak of leaf curling in chilli. In addition the presence of pesticide residues in chillies (Joia et al., 2001) ${ }^{[8]}$ has been of more concern for export of chillies to developed countries. In this context, it is therefore necessary to develop effective non-chemical pest management strategies against sucking pests for sustained crop management and production of healthy food. In view of this indiscriminate use of chemical pesticides and public concerns, the rise of new generation insecticides provides an alternative to reduce the ill effects of conventional insecticides. The pesticides molecules of new generation have been claimed to be effective as well as safer for non-target organism. Realizing serious pest status of the thrips, few promising, and widely recommended insecticides were incorporated in the present investigation.

\section{Materials and Methods}

The Experiments was carried out under field conditions at the Horticulture farm, Indira Gandhi Krishi Vishwavidyalaya,
Raipur, during Rabi 2017-18 \& 2018-19. Bio - efficacy of five insecticidal treatments comprising Emamectin Benzoate $3.5 \%$ + lambda cyhalothrin 5\% WP at three different doses viz.,11.00, 16.00 and $21.25 \mathrm{~g}$ a.i./ha respectively has been tested against thrips along with single dose of Emamectin Benzoate $5 \%$ SG (10 g a.i./ ha) and lambda cyhalothrin 5\% EC (15 g a.i./ ha) were sprayed with an untreated control check was shown in Randomized Block Design. There were three replications with $5 \mathrm{~m} \times 4 \mathrm{~m}$ plot size. The plant spacing between row to row and plant to plant was maintained $60 \mathrm{~cm}$ x $60 \mathrm{~cm}$, respectively.

The observations on total number of thrips, mite and aphid were recorded on top, middle and bottom leaves of five randomly selected plants from each treatment at one day before insecticide application while post-treatment observation made after 1, 3, 7, 10 and 15 days after spraying five plants randomly selected from each plot to work out the mean reduction in the insect population over control using statistical analysis.

Two sprays of each treatment were applied. Fruits were harvested from each plot separately and yield per plant each picking was recorded in $\mathrm{kg}$. Total yield was worked out by adding the yield of each picking. The yield per plot was converted to quintals per hectare.

\section{Treatments detail}

\begin{tabular}{|c|c|c|}
\hline S. No. & Treatments & Dose \\
\hline $\mathrm{T}_{1}$ & Emamectin Benzoate 3.5\% + lambda cyhalothrin 5\% WP & $11 \mathrm{~g}$ a.i./ha \\
\hline $\mathrm{T}_{2}$ & Emamectin Benzoate 3.5\% + lambda cyhalothrin 5\% WP & $16 \mathrm{~g} \mathrm{a}$.i.//ha \\
\hline $\mathrm{T}_{3}$ & Emamectin Benzoate 3.5\% + lambda cyhalothrin 5\% WP & $21.25 \mathrm{~g}$ a.i./ha \\
\hline $\mathrm{T}_{4}$ & Emamectin Benzoate 5\% SG & $10 \mathrm{~g}$ a.i.//ha \\
\hline $\mathrm{T}_{5}$ & Lambda cyhalothrin 5\% EC & $15 \mathrm{~g}$ a.i./ha \\
\hline $\mathrm{T}_{6}$ & Control (Untreated) & - \\
\hline
\end{tabular}

Table 1: Bio-efficacy of Emamectin benzoate 3.5\%+Lambda cyhalothrin 5\% WP against thrips on chilli pooled data during Rabi 2017-18 \& 2018-19

\begin{tabular}{|c|c|c|c|c|c|c|c|c|c|c|c|c|c|c|}
\hline \multirow{3}{*}{ Treatment } & \multirow{3}{*}{ Insecticide } & \multirow{3}{*}{ Dose } & \multirow{3}{*}{$\begin{array}{c}\text { Pre } \\
\text { treatment }\end{array}$} & \multicolumn{10}{|c|}{ Average no. of thrips/ plant } & \multirow{3}{*}{$\begin{array}{l}\text { Over } \\
\text { all } \\
\text { mean }\end{array}$} \\
\hline & & & & \multicolumn{5}{|c|}{ I Spray } & \multicolumn{5}{|c|}{ II Spray } & \\
\hline & & & & $\begin{array}{c}1 \\
\text { DAS }\end{array}$ & \begin{tabular}{|c|}
3 \\
DAS
\end{tabular} & $\begin{array}{c}7 \\
\text { DAS }\end{array}$ & $\begin{array}{c}10 \\
\text { DAS }\end{array}$ & $\begin{array}{c}15 \\
\text { DAS }\end{array}$ & \begin{tabular}{|c|}
1 \\
DAS
\end{tabular} & $\begin{array}{c}3 \\
\text { DAS }\end{array}$ & $\begin{array}{c}7 \\
\text { DAS }\end{array}$ & $\begin{array}{c}10 \\
\text { DAS }\end{array}$ & \begin{tabular}{|c|}
15 \\
DAS
\end{tabular} & \\
\hline $\mathrm{T}_{1}$ & $\begin{array}{c}\text { Emamectin Benzoate } \\
3.5 \% \text { + lambda } \\
\text { cyhalothrin 5\% WP }\end{array}$ & $\begin{array}{c}11 \\
\text { ga.i./ha }\end{array}$ & 24.81 & $\begin{array}{l}19.54 \\
(4.97)\end{array}$ & $\begin{array}{l}16.58 \\
(4.07)\end{array}$ & $\begin{array}{l}14.52 \\
(3.81)\end{array}$ & $\begin{array}{l}18.63 \\
(4.31)\end{array}$ & $\begin{array}{l}23.63 \\
(4.86)\end{array}$ & $\begin{array}{l}19.53 \\
(4.41)\end{array}$ & $\begin{array}{l}16.68 \\
(4.08)\end{array}$ & $\begin{array}{l}12.63 \\
(3.55)\end{array}$ & $\begin{array}{l}14.52 \\
(3.81)\end{array}$ & $\begin{array}{l}16.51 \\
(4.06)\end{array}$ & 17.96 \\
\hline $\mathrm{T}_{2}$ & $\begin{array}{c}\text { Emamectin Benzoate } \\
3.5 \% \text { + lambda } \\
\text { cyhalothrin 5\% WP }\end{array}$ & 16 & & $\begin{array}{l}13.54 \\
(4.24)\end{array}$ & $\begin{array}{l}10.58 \\
(3.25)\end{array}$ & $\begin{array}{l}12.52 \\
(3.53)\end{array}$ & $\begin{array}{l}18.61 \\
(4.31)\end{array}$ & $\begin{array}{l}21.63 \\
(4.65)\end{array}$ & $\begin{array}{l}11.53 \\
(3.39)\end{array}$ & $\begin{array}{l}10.68 \\
(3.26)\end{array}$ & $\begin{array}{c}9.63 \\
(3.10)\end{array}$ & $\begin{array}{l}10.58 \\
(3.25)\end{array}$ & $\begin{array}{l}15.51 \\
(3.93)\end{array}$ & 13.48 \\
\hline $\mathrm{T}_{3}$ & $\begin{array}{c}\text { Emamectin Benzoate } \\
3.5 \% \text { + lambda } \\
\text { cyhalothrin 5\% WP }\end{array}$ & $\begin{array}{c}21.25 \\
\text { ga.i./ha }\end{array}$ & & & $\begin{array}{l}11.55 \\
(3.39)\end{array}$ & $\begin{array}{c}8.53 \\
(2.92)\end{array}$ & $\begin{array}{l}11.55 \\
(3.39)\end{array}$ & $\begin{array}{l}15.52 \\
(3.94)\end{array}$ & $\begin{array}{c}7.53 \\
(2.74)\end{array}$ & $\begin{array}{c}4.51 \\
(2.12)\end{array}$ & $\begin{array}{c}2.55 \\
(1.59)\end{array}$ & $\begin{array}{c}5.53 \\
(2.35)\end{array}$ & $\begin{array}{c}7.55 \\
(2.74)\end{array}$ & 10.21 \\
\hline $\mathrm{T}_{4}$ & $\begin{array}{c}\text { Emamectin Benzoate } \\
5 \% \mathrm{SG}\end{array}$ & $\begin{array}{c}10 \\
\text { ga.i./ha }\end{array}$ & & .63 & \begin{tabular}{|l|}
19.58 \\
$(4.42)$
\end{tabular} & $\begin{array}{l}18.62 \\
(4.31)\end{array}$ & $\begin{array}{l}23.38 \\
(4.83)\end{array}$ & \begin{tabular}{|l|}
26.48 \\
$(5.14)$
\end{tabular} & $\begin{array}{l}23.37 \\
(4.83)\end{array}$ & \begin{tabular}{|l|}
21.27 \\
$(4.61)$
\end{tabular} & $\begin{array}{l}18.51 \\
(4.30)\end{array}$ & $\begin{array}{l}18.27 \\
(4.27)\end{array}$ & $\begin{array}{l}21.23 \\
(4.60)\end{array}$ & 21.61 \\
\hline $\mathrm{T}_{5}$ & $\begin{array}{c}\text { Lambda cyhalothrin 5\% } \\
\text { EC }\end{array}$ & $\begin{array}{l}15 \mathrm{ml} \\
\text { a.i./ha }\end{array}$ & $\begin{array}{l}25.36 \\
(5.58) \\
\end{array}$ & \begin{tabular}{|l|}
22.60 \\
$(4.75)$ \\
\end{tabular} & \begin{tabular}{|l|}
18.51 \\
$(4.30)$ \\
\end{tabular} & $\begin{array}{l}17.68 \\
(4.20)\end{array}$ & $\begin{array}{l}21.54 \\
(4.64)\end{array}$ & $\begin{array}{l}24.62 \\
(4.96)\end{array}$ & $\begin{array}{l}20.64 \\
(4.54)\end{array}$ & $\begin{array}{l}17.57 \\
(4.19)\end{array}$ & $\begin{array}{l}15.78 \\
(3.97)\end{array}$ & $\begin{array}{l}21.56 \\
(4.64)\end{array}$ & \begin{tabular}{|l|}
22.55 \\
$(4.74)$
\end{tabular} & 20.76 \\
\hline \multirow[t]{3}{*}{16} & Control (Untreated) & - & $\begin{array}{l}24.86 \\
(5.53)\end{array}$ & $\begin{array}{l}23.60 \\
(4.85)\end{array}$ & $\begin{array}{l}25.51 \\
(5.05)\end{array}$ & $\begin{array}{l}27.68 \\
(5.26)\end{array}$ & $\begin{array}{l}29.54 \\
(5.43)\end{array}$ & \begin{tabular}{|l|}
28.62 \\
$(5.35)$
\end{tabular} & \begin{tabular}{|l|}
30.64 \\
$(5.53)$
\end{tabular} & \begin{tabular}{|l|}
33.57 \\
$(5.79)$
\end{tabular} & $\begin{array}{l}35.78 \\
(5.98)\end{array}$ & \begin{tabular}{|l|}
37.56 \\
$(6.12)$
\end{tabular} & \begin{tabular}{|c|}
39.55 \\
$(6.28)$
\end{tabular} & 30.62 \\
\hline & \multirow{2}{*}{\multicolumn{2}{|c|}{ SEm \pm}} & 0.047 & 0.015 & 0.005 & 0.002 & 0.025 & 0.002 & 0.002 & 0.006 & 0.004 & 0.002 & 0.007 & \\
\hline & & & NS & 0.045 & 0.015 & 0.006 & 0.079 & 0.006 & 0.007 & 0.018 & 0.012 & 0.007 & 0.021 & \\
\hline
\end{tabular}

$\mathrm{DAS}=$ days after spray, ( ) figures in parentheses are square root transformed, $\mathrm{NS}=$ Non significant, $\mathrm{S}=$ significant. 


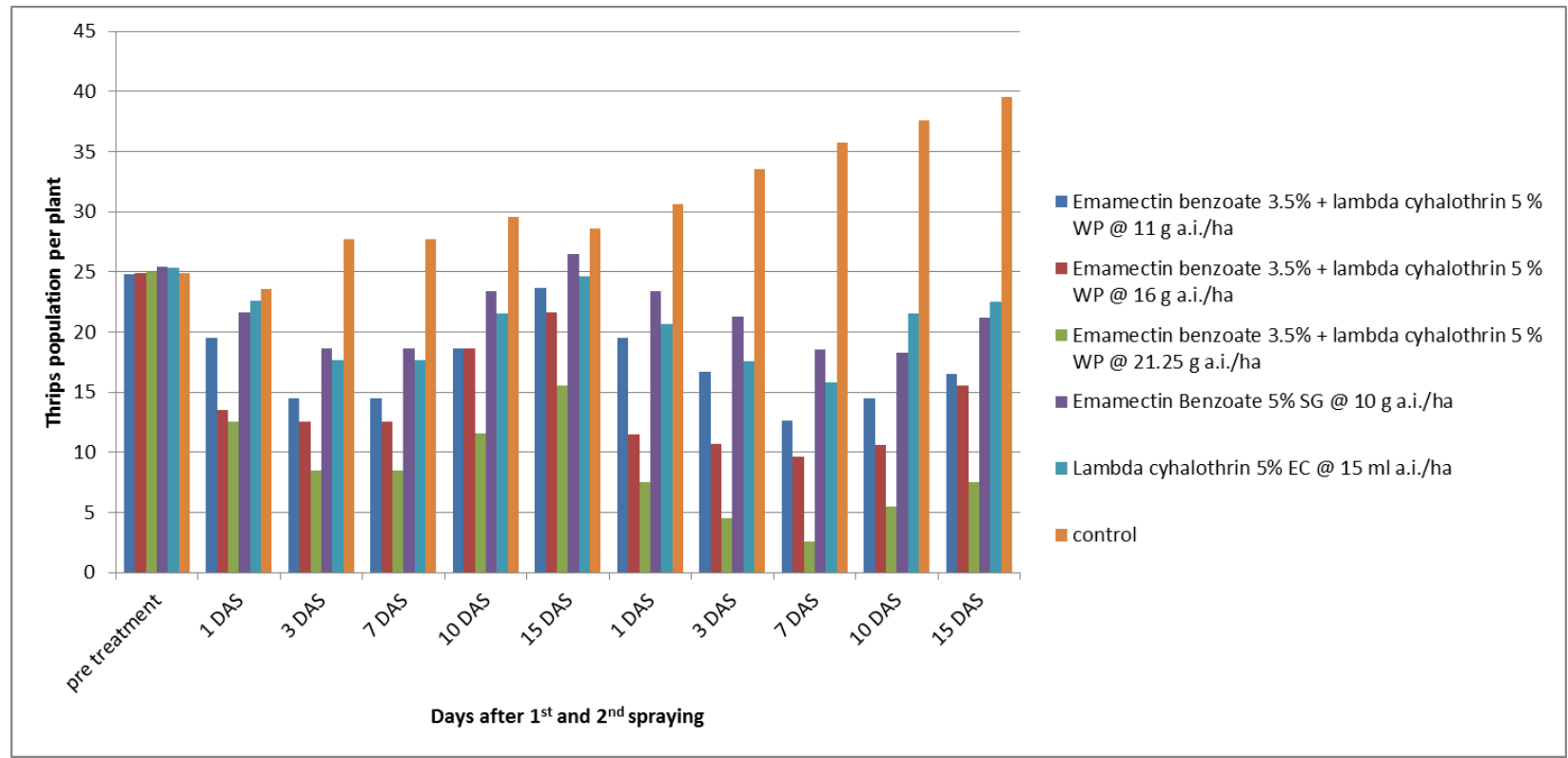

Fig 1: Bio-efficacy of Emamectin benzoate $3.5 \%+$ Lambda cyhalothrin 5\% WP against thrips on chilli after $1^{\text {st }} \& 2^{\text {nd }}$ spray.

Table 2: Bio-efficacy of Emamectin benzoate 3.5\% + Lambda cyhalothrin 5\% WP against mite on chilli pooled data during Rabi 2017-18 \& 2018-19

\begin{tabular}{|c|c|c|c|c|c|c|c|c|c|c|c|c|c|c|}
\hline \multirow{2}{*}{$\begin{array}{l}\text { Treat } \\
\text { ment }\end{array}$} & \multirow{2}{*}{ Insecticide } & \multirow{2}{*}{ dose } & \multirow{2}{*}{$\begin{array}{c}\text { Pre } \\
\text { treatment }\end{array}$} & \multicolumn{5}{|c|}{ I Spray } & \multicolumn{5}{|c|}{ II Spray } & \multirow{2}{*}{$\begin{array}{c}\text { Over all } \\
\text { mean }\end{array}$} \\
\hline & & & & 1DAS & 3DAS & 7DAS & 10DAS & 15DAS & 1DAS & 3DAS & 7DAS & 10DAS & 15DAS & \\
\hline $\mathrm{T}_{1}$ & $\begin{array}{c}\text { Emamectin Benzoate } \\
3.5 \%+\text { lambda } \\
\text { cyhalothrin 5\% WP }\end{array}$ & \begin{tabular}{|c|}
$11 \mathrm{~g}$ \\
a.i./ha
\end{tabular} & $3.78(1.94)$ & $\begin{array}{c}3.38 \\
(1.83)\end{array}$ & $\begin{array}{c}2.48 \\
(1.57)\end{array}$ & $\begin{array}{c}2.29 \\
(1.51)\end{array}$ & $\begin{array}{c}2.42 \\
(1.55)\end{array}$ & $\begin{array}{c}3.51 \\
(1.87)\end{array}$ & $\begin{array}{c}3.12 \\
(1.76)\end{array}$ & $\begin{array}{c}2.99 \\
(1.72)\end{array}$ & $\begin{array}{c}2.49 \\
(1.57)\end{array}$ & $\begin{array}{c}2.99 \\
(1.72)\end{array}$ & $\begin{array}{c}3.22 \\
(1.79)\end{array}$ & 2.97 \\
\hline $\mathrm{T}_{2}$ & $\begin{array}{c}\text { Emamectin Benzoate } \\
3.5 \% \text { + lambda } \\
\text { cyhalothrin 5\% WP }\end{array}$ & \begin{tabular}{|c|}
$16 \mathrm{~g}$ \\
a.i./ha
\end{tabular} & $4.00(2.00)$ & $\begin{array}{c}3.13 \\
(1.76)\end{array}$ & $\begin{array}{c}2.26 \\
(1.50)\end{array}$ & $\begin{array}{c}2.09 \\
(1.44)\end{array}$ & $\begin{array}{c}2.24 \\
(1.49)\end{array}$ & $\begin{array}{c}2.54 \\
(1.59)\end{array}$ & $\begin{array}{c}2.38 \\
(1.54)\end{array}$ & $\begin{array}{c}2.22 \\
(1.48)\end{array}$ & $\begin{array}{c}2.20 \\
(1.48)\end{array}$ & $\begin{array}{c}2.50 \\
(1.58)\end{array}$ & $\begin{array}{c}2.67 \\
(1.63)\end{array}$ & 2.53 \\
\hline $\mathrm{T}_{3}$ & $\begin{array}{c}\text { Emamectin Benzoate } \\
3.5 \%+\text { lambda } \\
\text { cyhalothrin 5\% WP }\end{array}$ & $\begin{array}{c}21.25 \mathrm{~g} \\
\text { a.i./ha }\end{array}$ & $3.08(1.75)$ & $\begin{array}{c}2.13 \\
(1.45)\end{array}$ & $\begin{array}{l}1.09 \\
(1.04)\end{array}$ & $\begin{array}{c}1.39 \\
(1.17)\end{array}$ & $\begin{array}{c}1.65 \\
(1.28)\end{array}$ & $\begin{array}{l}2.43 \\
(1.55)\end{array}$ & $\begin{array}{c}2.03 \\
(1.42)\end{array}$ & $\begin{array}{l}1.55 \\
(1.24)\end{array}$ & $\begin{array}{c}2.10 \\
(1.44)\end{array}$ & $\begin{array}{c}2.46 \\
(1.56)\end{array}$ & $\begin{array}{l}2.60 \\
(1.61)\end{array}$ & 2.04 \\
\hline $\mathrm{T}_{4}$ & $\begin{array}{c}\text { Emamectin Benzoate 5\% } \\
\text { SG }\end{array}$ & \begin{tabular}{|c|}
$10 \mathrm{~g}$ \\
a.i./ha
\end{tabular} & $4.94(2.22)$ & \begin{tabular}{|c|}
3.77 \\
$(1.94)$
\end{tabular} & $\begin{array}{c}3.51 \\
(1.87)\end{array}$ & \begin{tabular}{|c|}
3.39 \\
$(1.84)$
\end{tabular} & $\begin{array}{c}3.65 \\
(1.91)\end{array}$ & $\begin{array}{c}3.82 \\
(1.95)\end{array}$ & \begin{tabular}{|c|}
3.65 \\
$(1.91)$
\end{tabular} & $\begin{array}{c}3.56 \\
(1.88)\end{array}$ & $\begin{array}{c}3.46 \\
(1.86)\end{array}$ & $\begin{array}{c}3.75 \\
(1.93)\end{array}$ & $\begin{array}{c}3.83 \\
(1.95)\end{array}$ & 3.66 \\
\hline $\mathrm{T}_{5}$ & $\begin{array}{c}\text { Lambda cyhalothrin 5\% } \\
\text { EC }\end{array}$ & \begin{tabular}{|l|}
$15 \mathrm{ml}$ \\
a.i./ha \\
\end{tabular} & $5.82(2.41)$ & $\begin{array}{c}3.54 \\
(1.88) \\
\end{array}$ & $\begin{array}{c}3.34 \\
(1.82) \\
\end{array}$ & $\begin{array}{c}3.21 \\
(1.79) \\
\end{array}$ & $\begin{array}{c}3.31 \\
(1.81) \\
\end{array}$ & $\begin{array}{c}3.41 \\
(1.84) \\
\end{array}$ & \begin{tabular}{|c|}
3.15 \\
$(1.77)$ \\
\end{tabular} & $\begin{array}{c}3.03 \\
(1.74) \\
\end{array}$ & \begin{tabular}{|c|}
2.88 \\
$(1.69)$ \\
\end{tabular} & $\begin{array}{c}3.12 \\
(1.76) \\
\end{array}$ & $\begin{array}{c}3.19 \\
(1.78) \\
\end{array}$ & 3.27 \\
\hline $\mathrm{T}_{6}$ & Control (Untreated) & - & $3.85(1.96)$ & $\begin{array}{c}3.81 \\
(1.95)\end{array}$ & $\begin{array}{c}4.85 \\
(2.20)\end{array}$ & $\begin{array}{c}6.38 \\
(2.52)\end{array}$ & $\begin{array}{c}5.40 \\
(2.32)\end{array}$ & $\begin{array}{c}7.42 \\
(2.72)\end{array}$ & $\begin{array}{c}6.44 \\
(2.53)\end{array}$ & $\begin{array}{c}6.46 \\
(2.54)\end{array}$ & $\begin{array}{c}6.48 \\
(2.54)\end{array}$ & $\begin{array}{l}6.50 \\
(2.55)\end{array}$ & $\begin{array}{l}7.52 \\
(2.74)\end{array}$ & 5.92 \\
\hline & SEm \pm & - & 1.08 & 1.01 & 1.03 & 1.03 & 1.03 & 1.01 & 1.03 & 1.04 & 1.03 & 1.06 & 1.06 & \\
\hline & $\mathrm{CD}$ at $5 \%$ & - & $\mathrm{NS}$ & 1.13 & 1.12 & 1.13 & 1.15 & 1.07 & 1.13 & 1.14 & 1.13 & 1.23 & 1.23 & \\
\hline
\end{tabular}

DAS= days after spray, ( ) figures in parentheses are square root transformed, NS= Non significant, $\mathrm{S}=$ significant.

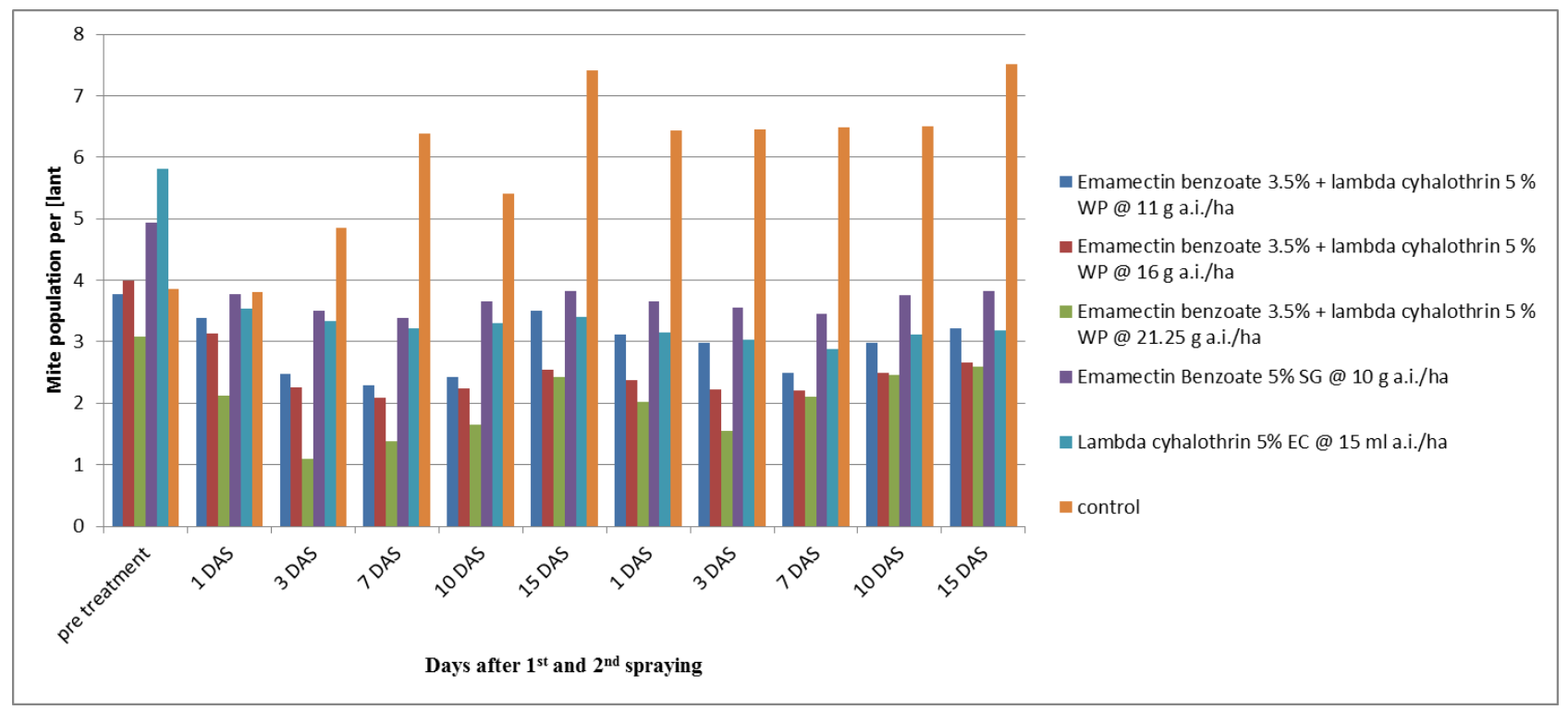

Fig 2: Bio-efficacy of Emamectin benzoate 3.5\% + Lambda cyhalothrin 5\% WP against mite on chilli after $1^{\text {st }} \& 2^{\text {nd }}$ spray. 
Table 3: Bio-efficacy of Emamectin benzoate 3.5\% + Lambda cyhalothrin 5\% WP against aphid on chilli pooled data during Rabi 2017-18 and 2018-19

\begin{tabular}{|c|c|c|c|c|c|c|c|c|c|c|c|c|c|c|}
\hline \multirow{2}{*}{ Treatment } & \multirow{2}{*}{ Insecticide } & \multirow{2}{*}{ Dose } & \multirow{2}{*}{\begin{tabular}{|c|}
$\begin{array}{c}\text { Pre } \\
\text { treatment }\end{array}$ \\
\end{tabular}} & \multicolumn{5}{|c|}{$1^{\text {st }}$ Spray } & \multicolumn{5}{|c|}{$2^{\text {nd }}$ Spray } & \multirow{2}{*}{\begin{tabular}{|c} 
Overall \\
Mean \\
\end{tabular}} \\
\hline & & & & 1DAS & 3DAS & 7DAS & 10DAS & 15DAS & 1DAS & 3DAS & 7DAS & 10DAS & 15DAS & \\
\hline $\mathrm{T} 1$ & $\begin{array}{c}\text { Emamectin Benzoate } \\
3.5 \%+\text { lambda } \\
\text { cyhalothrin 5\% WP }\end{array}$ & \begin{tabular}{|l}
$11 \mathrm{~g}$ \\
a.i./ha
\end{tabular} & $6.69(3.18)$ & $\begin{array}{c}4.28 \\
(2.68)\end{array}$ & $\begin{array}{c}4.79 \\
(2.80)\end{array}$ & $\begin{array}{c}5.62 \\
(2.97)\end{array}$ & $\begin{array}{c}6.59 \\
(3.16)\end{array}$ & $\begin{array}{c}6.66 \\
(3.17)\end{array}$ & $\begin{array}{l}3.72 \\
(5.1)\end{array}$ & $\begin{array}{c}3.92 \\
(2.60)\end{array}$ & $\begin{array}{c}4.22 \\
(2.67)\end{array}$ & $\begin{array}{c}4.62 \\
(2.75)\end{array}$ & $\begin{array}{c}5.12 \\
(2.86)\end{array}$ & 5.11 \\
\hline $\mathrm{T} 2$ & $\begin{array}{c}\text { Emamectin Benzoate } \\
3.5 \%+\text { lambda } \\
\text { cyhalothrin 5\% WP } \\
\end{array}$ & $\begin{array}{c}16 \mathrm{~g} \\
\text { a.i./ha }\end{array}$ & $6.43(3.12)$ & $\begin{array}{l}3.15 \\
(2.41)\end{array}$ & $\begin{array}{c}3.82 \\
(2.57)\end{array}$ & $\begin{array}{c}4.85 \\
(2.81)\end{array}$ & $\begin{array}{c}5.52 \\
(2.95)\end{array}$ & $\begin{array}{c}6.38 \\
(3.12)\end{array}$ & $\begin{array}{c}3.22 \\
(2.42)\end{array}$ & $\begin{array}{c}3.42 \\
(2.47)\end{array}$ & $\begin{array}{c}3.72 \\
(2.54)\end{array}$ & $\begin{array}{l}4.12 \\
(2.64)\end{array}$ & $\begin{array}{c}4.62 \\
(2.94)\end{array}$ & 4.47 \\
\hline $\mathrm{T} 3$ & $\begin{array}{c}\text { Emamectin Benzoate } \\
3.5 \% \text { + lambda } \\
\text { cyhalothrin 5\% WP }\end{array}$ & $\begin{array}{l}21.25 \mathrm{~g} \\
\text { a.i./ha }\end{array}$ & $6.47(3.13)$ & $\begin{array}{c}2.14 \\
(2.12)\end{array}$ & $\begin{array}{c}2.92 \\
(2.32)\end{array}$ & $\begin{array}{c}3.95 \\
(2.60)\end{array}$ & $\begin{array}{c}4.52 \\
(2.73)\end{array}$ & $\begin{array}{c}5.52 \\
(3.95)\end{array}$ & $\begin{array}{c}2.02 \\
(1.98)\end{array}$ & $\begin{array}{c}2.23 \\
(2.14)\end{array}$ & $\begin{array}{c}2.52 \\
(2.23)\end{array}$ & $\begin{array}{c}2.92 \\
(2.34)\end{array}$ & $\begin{array}{c}3.42 \\
(2.46)\end{array}$ & 3.51 \\
\hline $\mathrm{T} 4$ & $\begin{array}{c}\text { Emamectin Benzoate } \\
5 \% \mathrm{SG} \\
\end{array}$ & \begin{tabular}{|c|}
$10 \mathrm{~g}$ \\
a.i./ha \\
\end{tabular} & $6.62(3.17)$ & \begin{tabular}{|c|}
6.29 \\
$(3.10)$ \\
\end{tabular} & \begin{tabular}{|c|}
6.33 \\
$(3.11)$ \\
\end{tabular} & \begin{tabular}{|c|}
6.49 \\
$(3.14)$ \\
\end{tabular} & $\begin{array}{c}6.53 \\
(3.15) \\
\end{array}$ & $\begin{array}{c}7.36 \\
(3.30)\end{array}$ & \begin{tabular}{|c|}
5.36 \\
$(3.30)$ \\
\end{tabular} & \begin{tabular}{|c|}
5.56 \\
$(2.96)$ \\
\end{tabular} & $\begin{array}{c}5.86 \\
(3.02) \\
\end{array}$ & $\begin{array}{c}6.26 \\
(3.10) \\
\end{array}$ & \begin{tabular}{|c|}
6.76 \\
$(3.19)$ \\
\end{tabular} & 6.31 \\
\hline T5 & $\begin{array}{c}\text { Lambda cyhalothrin } \\
5 \% \mathrm{EC} \\
\end{array}$ & \begin{tabular}{|l|}
$15 \mathrm{ml}$ \\
a.i./ha \\
\end{tabular} & 6.59 (3.16) & $\begin{array}{c}5.16 \\
(2.87) \\
\end{array}$ & \begin{tabular}{|c|}
5.99 \\
$(3.04)$ \\
\end{tabular} & $\begin{array}{c}6.46 \\
(3.12) \\
\end{array}$ & $\begin{array}{c}6.69 \\
(3.18)\end{array}$ & $\begin{array}{c}7.22 \\
(3.28)\end{array}$ & $\begin{array}{c}4.32 \\
(2.69)\end{array}$ & \begin{tabular}{|c|}
4.52 \\
$(2.74)$ \\
\end{tabular} & $\begin{array}{l}4.82 \\
(2.8)\end{array}$ & $\begin{array}{c}5.22 \\
(2.89)\end{array}$ & $\begin{array}{c}5.72 \\
(2.99)\end{array}$ & 5.70 \\
\hline T6 & Control (Untreated) & - & $6.49(3.12)$ & $\begin{array}{c}6.63 \\
(3.16)\end{array}$ & $\begin{array}{c}6.96 \\
(3.23)\end{array}$ & $\begin{array}{c}7.99 \\
(3.41)\end{array}$ & $\begin{array}{c}8.66 \\
(3.52)\end{array}$ & $\begin{array}{c}9.43 \\
(3.65)\end{array}$ & $\begin{array}{c}9.86 \\
(3.71)\end{array}$ & $\begin{array}{l}10.53 \\
(3.82)\end{array}$ & $\begin{array}{l}11.36 \\
(3.94)\end{array}$ & $\begin{array}{l}11.53 \\
(3.95)\end{array}$ & $\begin{array}{l}12.26 \\
(4.06)\end{array}$ & 9.24 \\
\hline & \multicolumn{2}{|l|}{ SEm +} & 0.029 & 0.032 & 0.026 & 0.033 & 0.040 & 0.041 & 0.032 & 0.023 & 0.018 & 0.036 & 0.018 & \\
\hline & \multicolumn{2}{|l|}{$\mathrm{CD}$ at $5 \%$} & NS & 0.102 & 0.082 & 0.106 & 0.127 & 0.132 & 0.103 & 0.073 & 0.058 & 0.114 & 0.058 & \\
\hline
\end{tabular}

$\mathrm{DAS}=$ days after spray, ( ) figures in parentheses are square root transformed, NS= Non significant, $\mathrm{S}=$ significant.

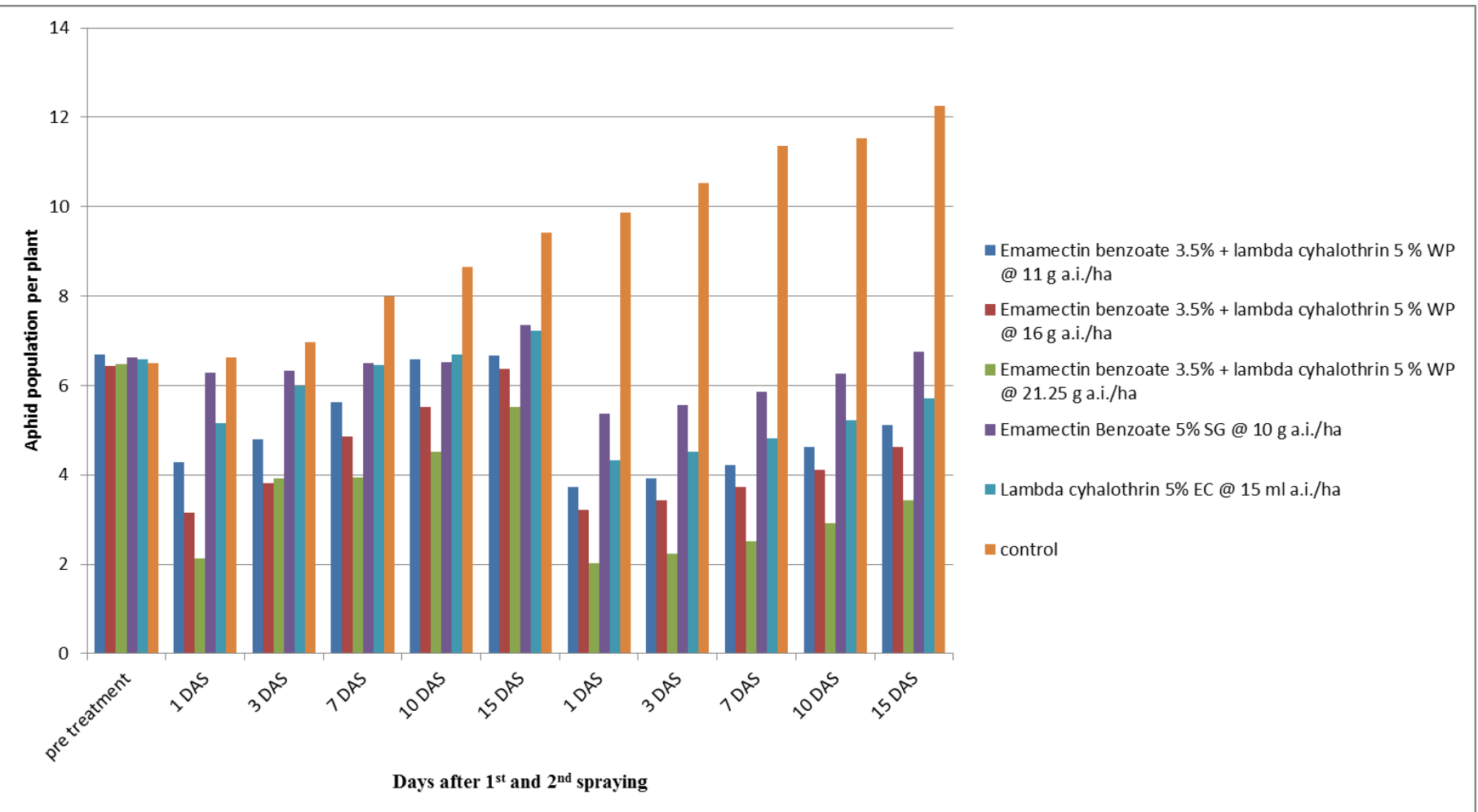

Fig. 3: Bio-efficacy of Emamectin benzoate $3.5 \%+$ Lambda cyhalothrin 5\% WP against aphids on chilli after $1^{\text {st }} \& 2^{\text {nd }}$ spray

Table 4: Effect of Emamectin benzoate 3.5\%+Lambda cyhalothrin 5\% WP against Coccinellids on chilli pooled data during Rabi 2017-18 \& 2018-19

\begin{tabular}{|c|c|c|c|c|c|c|c|c|c|c|c|c|c|c|}
\hline \multirow[t]{2}{*}{ Treatment } & \multirow[t]{2}{*}{ Insecticide } & \multirow[t]{2}{*}{ dose } & \multirow{2}{*}{$\begin{array}{c}\text { Pre } \\
\text { treatment }\end{array}$} & \multicolumn{5}{|c|}{ I Spray } & \multicolumn{5}{|c|}{ II Spray } & \multirow[t]{2}{*}{$\begin{array}{c}\text { Over } \\
\text { all } \\
\text { mean }\end{array}$} \\
\hline & & & & 1 DAS & 3DAS & 7DAS & 10DAS & 15DAS & 1 DAS & 3DAS & 7DAS & 10DAS & 15DAS & \\
\hline $\mathrm{T}_{1}$ & $\begin{array}{c}\text { Emamectin Benzoate } \\
3.5 \% \text { + lambda } \\
\text { cyhalothrin 5\% WP }\end{array}$ & $\begin{array}{l}11 \mathrm{~g} \\
\text { a.i./ha }\end{array}$ & $\begin{array}{c}0.46 \\
(1.36)\end{array}$ & $\left|\begin{array}{c}0.3 \\
(1.25)\end{array}\right|$ & $\begin{array}{c}0.46 \\
(1.36)\end{array}$ & $\begin{array}{c}0.28 \\
(1.18)\end{array}$ & $\begin{array}{c}0.42 \\
(1.32)\end{array}$ & $\begin{array}{c}0.43 \\
(1.33)\end{array}$ & $\begin{array}{c}0.51 \\
(1.41)\end{array}$ & $\begin{array}{c}0.43 \\
(1.33)\end{array}$ & $\begin{array}{c}0.45 \\
(1.35)\end{array}$ & $\begin{array}{c}0.46 \\
(1.36)\end{array}$ & $\begin{array}{c}0.40 \\
(1.30)\end{array}$ & 0.43 \\
\hline $\mathrm{T}_{2}$ & $\begin{array}{c}\text { Emamectin Benzoate } \\
3.5 \% \text { + lambda } \\
\text { cyhalothrin 5\% WP }\end{array}$ & $\begin{array}{l}16 \mathrm{~g} \\
\text { a.i./ha }\end{array}$ & $\begin{array}{c}0.44 \\
(1.31)\end{array}$ & $\left|\begin{array}{c}0.41 \\
(1.31)\end{array}\right|$ & $\begin{array}{c}0.22 \\
(1.12)\end{array}$ & $\begin{array}{c}0.46 \\
(1.36)\end{array}$ & $\begin{array}{c}0.45 \\
(1.35)\end{array}$ & $\begin{array}{c}0.44 \\
(1.34)\end{array}$ & $\begin{array}{c}0.42 \\
(1.32)\end{array}$ & $\begin{array}{c}0.40 \\
(1.30)\end{array}$ & $\begin{array}{c}0.44 \\
(1.31)\end{array}$ & $\begin{array}{c}0.40 \\
(1.30)\end{array}$ & $\begin{array}{c}0.43 \\
(1.33)\end{array}$ & 0.41 \\
\hline $\mathrm{T}_{3}$ & $\begin{array}{c}\text { Emamectin Benzoate } \\
3.5 \%+\text { lambda } \\
\text { cyhalothrin 5\% WP }\end{array}$ & $\begin{array}{l}21.25 \mathrm{~g} \\
\text { a.i./ha }\end{array}$ & $\begin{array}{c}0.47 \\
(1.37)\end{array}$ & $\begin{array}{c}0.35 \\
(1.25)\end{array}$ & $\begin{array}{c}0.27 \\
(1.17)\end{array}$ & $\begin{array}{c}0.35 \\
(1.25)\end{array}$ & $\begin{array}{c}0.44 \\
(1.34)\end{array}$ & $\begin{array}{c}0.42 \\
(1.32)\end{array}$ & $\begin{array}{c}0.40 \\
(1.30)\end{array}$ & $\begin{array}{c}0.44 \\
(1.34)\end{array}$ & $\begin{array}{c}0.48 \\
(1.38)\end{array}$ & $\begin{array}{c}0.40 \\
(1.30)\end{array}$ & $\begin{array}{c}0.42 \\
(1.32)\end{array}$ & 0.42 \\
\hline $\mathrm{T}_{4}$ & $\begin{array}{c}\text { Emamectin Benzoate } \\
5 \% \mathrm{SG} \\
\end{array}$ & $\begin{array}{c}10 \mathrm{~g} \\
\text { a.i.//ha }\end{array}$ & $\begin{array}{c}0.42 \\
(1.32) \\
\end{array}$ & \begin{tabular}{|c|}
0.33 \\
$(1.23)$ \\
\end{tabular} & \begin{tabular}{|c|}
0.26 \\
$(1.16)$ \\
\end{tabular} & \begin{tabular}{|c|}
0.34 \\
$(1.24)$ \\
\end{tabular} & $\begin{array}{c}0.42 \\
(1.32) \\
\end{array}$ & $\begin{array}{c}0.43 \\
(1.33) \\
\end{array}$ & \begin{tabular}{|c|}
0.46 \\
$(1.36)$ \\
\end{tabular} & \begin{tabular}{|c|}
0.41 \\
$(1.31)$ \\
\end{tabular} & \begin{tabular}{|c|}
0.45 \\
$(1.31)$ \\
\end{tabular} & \begin{tabular}{|c|}
0.38 \\
$(1.28)$ \\
\end{tabular} & $\begin{array}{c}0.40 \\
(1.30) \\
\end{array}$ & 0.39 \\
\hline
\end{tabular}




\begin{tabular}{|c|c|c|c|c|c|c|c|c|c|c|c|c|c|c|}
\hline $\mathrm{T}_{5}$ & $\begin{array}{c}\text { Lambda cyhalothrin } 5 \% \\
\text { EC }\end{array}$ & $\begin{array}{c}15 \\
\mathrm{ml} \\
\text { a.i./ha }\end{array}$ & $\begin{array}{c}0.45 \\
(1.35)\end{array}$ & $\begin{array}{c}0.38 \\
(1.28)\end{array}$ & $\begin{array}{c}0.24 \\
(1.14)\end{array}$ & $\begin{array}{c}0.40 \\
(1.30)\end{array}$ & $\begin{array}{c}0.46 \\
(1.36)\end{array}$ & $\begin{array}{c}0.44 \\
(1.34)\end{array}$ & $\begin{array}{c}0.45 \\
(1.35)\end{array}$ & $\begin{array}{c}0.42 \\
(1.32)\end{array}$ & $\begin{array}{c}0.43 \\
(1.33)\end{array}$ & $\begin{array}{c}0.40 \\
(1.30)\end{array}$ & $\begin{array}{c}0.42 \\
(1.32)\end{array}$ & 0.40 \\
\hline $\mathrm{T}_{6}$ & Control & - & $\begin{array}{c}0.46 \\
(1.36)\end{array}$ & \begin{tabular}{|c|}
0.47 \\
$(1.37)$ \\
\end{tabular} & \begin{tabular}{|c|}
0.46 \\
$(1.36)$ \\
\end{tabular} & $\begin{array}{c}0.49 \\
(1.39)\end{array}$ & $\begin{array}{c}0.44 \\
(1.34)\end{array}$ & $\begin{array}{c}0.45 \\
(1.35)\end{array}$ & $\begin{array}{c}0.46 \\
(1.36)\end{array}$ & $\begin{array}{c}0.47 \\
(1.37)\end{array}$ & $\begin{array}{c}0.48 \\
(1.38)\end{array}$ & $\begin{array}{c}0.47 \\
(1.37)\end{array}$ & $\begin{array}{c}0.46 \\
(1.36)\end{array}$ & 0.46 \\
\hline & SEm \pm & & 0.21 & 0.21 & 0.01 & 0.21 & 0.21 & 0.21 & 0.21 & 0.21 & 0.21 & 0.21 & 0.21 & \\
\hline & $\mathrm{CD}$ at $5 \%$ & & NS & NS & NS & NS & NS & NS & NS & NS & $\mathrm{NS}$ & NS & NS & \\
\hline
\end{tabular}

DAS = days after spray, ( ) figures in parentheses are square root transformed, NS = Non significant, $\mathrm{S}=$ significant.

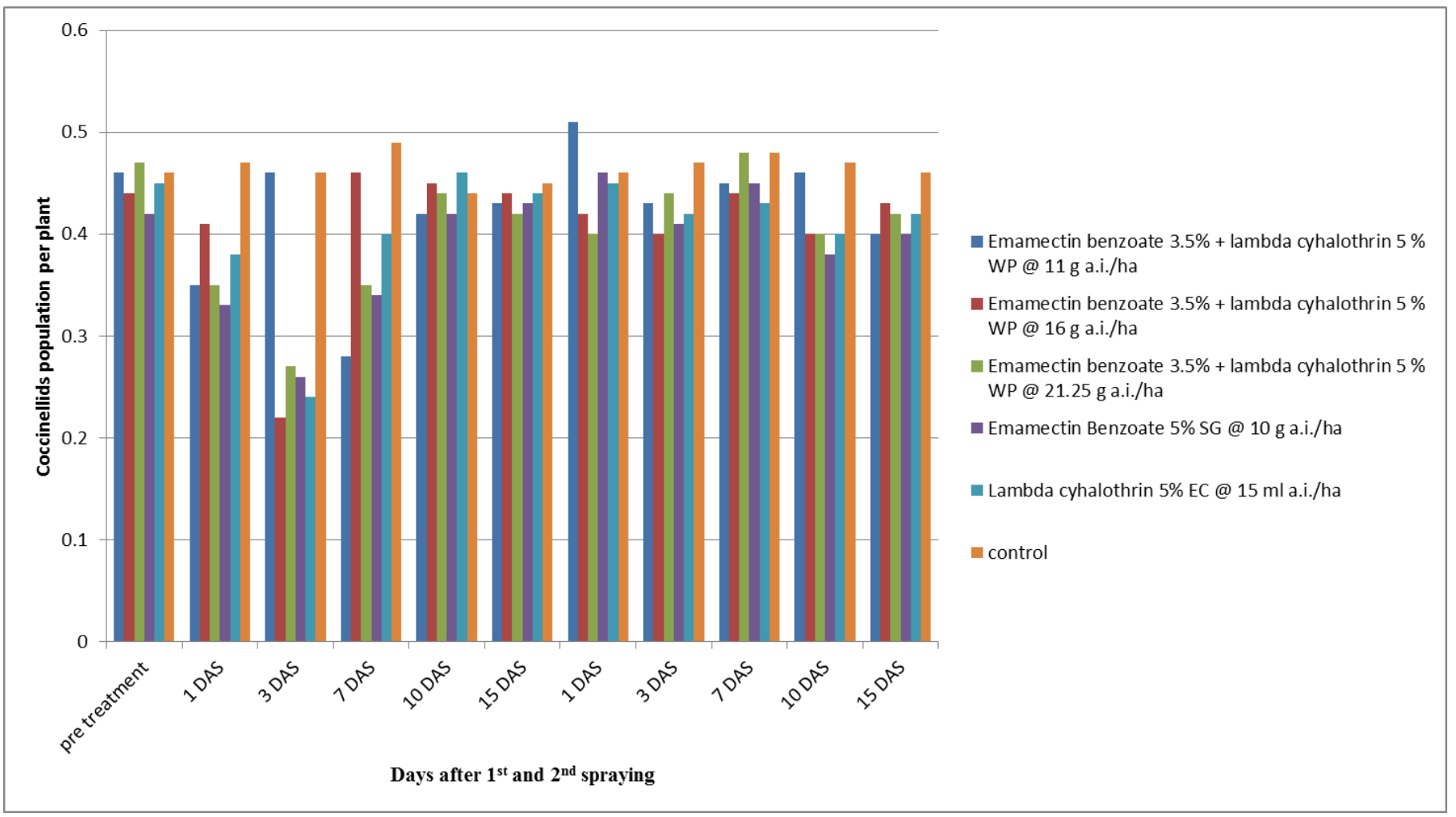

Fig. 4: Influence of Emamectin benzoate 3.5\% + Lambda cyhalothrin 5\% WP on the occurrence of coccinellids on chilli

Table 5: Effect of Emamectin benzoate 3.5\%+Lambda cyhalothrin 5\% WP against Spider on chilli pooled data during Rabi 2017-18 \& 2018-19

\begin{tabular}{|c|c|c|c|c|c|c|c|c|c|c|c|c|c|c|}
\hline \multirow{2}{*}{ Treatment } & \multirow{2}{*}{ Insecticide } & \multirow{2}{*}{ dose } & \multirow{2}{*}{$\begin{array}{c}\text { Pre } \\
\text { treatment }\end{array}$} & \multicolumn{5}{|c|}{ I Spray } & \multicolumn{5}{|c|}{ II Spray } & \multirow{2}{*}{$\begin{array}{c}\text { Over } \\
\text { all } \\
\text { mean }\end{array}$} \\
\hline & & & & $1 \mathrm{DAS}$ & 3DAS & 7DAS & 10DAS & 15DAS & 1 DAS & 3DAS & 7DAS & 10DAS & 15DAS & \\
\hline $\mathrm{T}_{1}$ & $\begin{array}{c}\text { Emamectin Benzoate } \\
3.5 \% \text { + lambda } \\
\text { cyhalothrin 5\% WP }\end{array}$ & $\begin{array}{l}11 \mathrm{~g} \\
\text { a.i./ha }\end{array}$ & $0.78(1.68)$ & $\begin{array}{c}0.68 \\
(1.58)\end{array}$ & $\begin{array}{c}0.72 \\
(1.62)\end{array}$ & $\mid \begin{array}{c}0.68 \\
(1.58)\end{array}$ & $\begin{array}{l}0.65 \\
(1.55)\end{array}$ & $\begin{array}{c}0.68 \\
(1.58)\end{array}$ & $\begin{array}{c}0.67 \\
(1.57)\end{array}$ & $\begin{array}{c}0.65 \\
(1.55)\end{array}$ & $\begin{array}{c}0.68 \\
(1.58)\end{array}$ & $\begin{array}{c}0.69 \\
(1.59)\end{array}$ & $\begin{array}{c}0.68 \\
(1.58)\end{array}$ & 0.67 \\
\hline $\mathrm{T}_{2}$ & $\begin{array}{c}\text { Emamectin Benzoate } \\
3.5 \% \text { + lambda } \\
\text { cyhalothrin 5\% WP }\end{array}$ & $\begin{array}{c}16 \mathrm{~g} \\
\text { a.i./ha }\end{array}$ & $0.68(1.58)$ & $\begin{array}{c}0.66 \\
(1.56)\end{array}$ & $\begin{array}{c}0.62 \\
(1.52)\end{array}$ & $\begin{array}{c}0.60 \\
(1.50)\end{array}$ & $\begin{array}{c}0.72 \\
(1.62)\end{array}$ & $\begin{array}{c}0.74 \\
(1.64)\end{array}$ & $\begin{array}{c}0.65 \\
(1.55)\end{array}$ & $\begin{array}{c}0.63 \\
(1.53)\end{array}$ & $\begin{array}{c}0.64 \\
(1.54)\end{array}$ & $\begin{array}{c}0.68 \\
(1.58)\end{array}$ & $\begin{array}{c}0.70 \\
(1.60)\end{array}$ & 0.65 \\
\hline $\mathrm{T}_{3}$ & $\begin{array}{c}\text { Emamectin Benzoate } \\
3.5 \%+\text { lambda } \\
\text { cyhalothrin 5\% WP }\end{array}$ & $\begin{array}{c}21.25 \mathrm{~g} \\
\text { a.i./ha }\end{array}$ & $0.66(1.56)$ & $\begin{array}{c}0.55 \\
(1.45)\end{array}$ & $\begin{array}{c}0.68 \\
(1.58)\end{array}$ & $\begin{array}{c}0.70 \\
(1.60)\end{array}$ & $\begin{array}{c}0.64 \\
(1.54)\end{array}$ & $\begin{array}{c}0.72 \\
(1.62)\end{array}$ & $\begin{array}{c}0.64 \\
(1.54)\end{array}$ & $\begin{array}{c}0.67 \\
(1.57)\end{array}$ & $\begin{array}{c}0.59 \\
(1.49)\end{array}$ & $\begin{array}{c}0.57 \\
(1.47)\end{array}$ & $\begin{array}{c}0.65 \\
(1.55)\end{array}$ & 0.64 \\
\hline $\mathrm{T}_{4}$ & $\begin{array}{c}\text { Emamectin Benzoate } \\
5 \% \mathrm{SG}\end{array}$ & $\begin{array}{l}10 \mathrm{~g} \\
\text { a.i./ha }\end{array}$ & $0.66(1.56)$ & $\begin{array}{c}0.64 \\
(1.54)\end{array}$ & $\begin{array}{c}0.62 \\
(1.52)\end{array}$ & \begin{tabular}{|c|}
0.63 \\
$(1.53)$
\end{tabular} & $\begin{array}{c}0.64 \\
(1.54)\end{array}$ & $\begin{array}{c}0.68 \\
(1.58)\end{array}$ & \begin{tabular}{|c|}
0.64 \\
$(1.54)$
\end{tabular} & $\begin{array}{c}0.62 \\
(1.52)\end{array}$ & $\begin{array}{c}0.60 \\
(1.50)\end{array}$ & $\begin{array}{c}0.61 \\
(1.51)\end{array}$ & $\begin{array}{c}0.64 \\
(1.54)\end{array}$ & 0.63 \\
\hline $\mathrm{T}_{5}$ & $\begin{array}{c}\text { Lambda cyhalothrin } \\
5 \% \text { EC }\end{array}$ & \begin{tabular}{|c|}
15 \\
mla.i./ha
\end{tabular} & $0.66(1.56)$ & $\begin{array}{c}0.55 \\
(1.45)\end{array}$ & $\begin{array}{c}0.48 \\
(1.38)\end{array}$ & $\begin{array}{c}0.45 \\
(1.35) \\
\end{array}$ & $\begin{array}{c}0.64 \\
(1.54)\end{array}$ & $\begin{array}{c}0.72 \\
(1.62)\end{array}$ & $\begin{array}{c}0.64 \\
(1.54) \\
\end{array}$ & $\begin{array}{c}0.62 \\
(1.52)\end{array}$ & $\begin{array}{c}0.59 \\
(1.49)\end{array}$ & $\begin{array}{c}0.63 \\
(1.53)\end{array}$ & $\begin{array}{c}0.65 \\
(1.55)\end{array}$ & 0.60 \\
\hline $\mathrm{T}_{6}$ & Control (Untreated) & - & $0.65(1.55)$ & $\begin{array}{c}0.68 \\
(1.58)\end{array}$ & $\begin{array}{c}0.69 \\
(1.59)\end{array}$ & $\begin{array}{c}0.70 \\
(1.60)\end{array}$ & $\begin{array}{c}0.71 \\
(1.61)\end{array}$ & $\begin{array}{c}0.72 \\
(1.62) \\
\end{array}$ & $\begin{array}{c}0.73 \\
(1.63) \\
\end{array}$ & $\begin{array}{c}0.72 \\
(1.62)\end{array}$ & $\begin{array}{c}0.73 \\
(1.63)\end{array}$ & $\begin{array}{c}0.71 \\
(1.61)\end{array}$ & $\begin{array}{c}0.72 \\
(1.61)\end{array}$ & 0.70 \\
\hline & $\mathrm{SEm} \pm$ & & 0.42 & 0.44 & 0.42 & 0.42 & 0.41 & 0.41 & 0.41 & 0.41 & 0.42 & 0.42 & 0.42 & \\
\hline & $\mathrm{CD}$ at $5 \%$ & & NS & NS & NS & NS & NS & NS & NS & NS & NS & NS & NS & \\
\hline
\end{tabular}

DAS= days after spray, ( ) figures in parentheses are square root transformed, NS= Non significant, $\mathrm{S}=$ significant. 


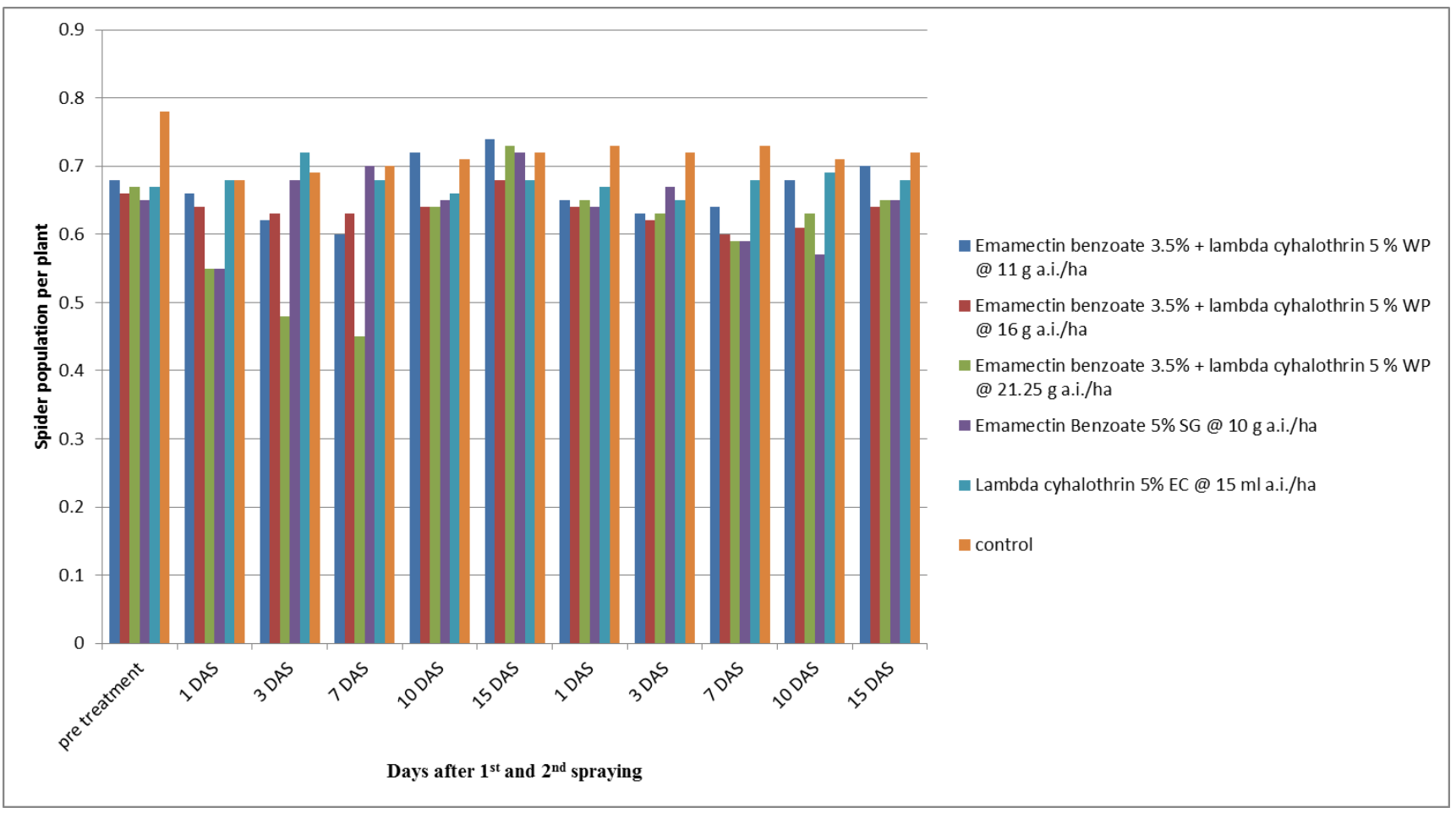

Fig. 5: Influence of Emamectin benzoate 3.5\% + Lambda cyhalothrin 5\% WP on the occurrence of spiders on chilli

Table 6: Mean yield data of chilli to application of different insecticides against different insect pest of chilli crops during Rabi 2017-18

\begin{tabular}{|c|c|c|c|}
\hline S. No. & Name of treatment & Dose & Green Chilli Yield (q/ha) \\
\hline T1 & Emamectin Benzoate 3.5\% + lambda cyhalothrin 5\% WP & $11 \mathrm{~g} \mathrm{a.i} . / \mathrm{ha}$ & 95.79 \\
\hline T2 & Emamectin Benzoate 3.5\% + lambda cyhalothrin 5\% WP & $16 \mathrm{~g}$ a.i./ha & 99.13 \\
\hline T3 & Emamectin Benzoate 3.5\% + lambda cyhalothrin 5\% WP & $21.25 \mathrm{~g}$ a.i./ha & 104.51 \\
\hline T4 & Emamectin Benzoate 5\% SG & $10 \mathrm{~g}$ a.i./ha & 83.02 \\
\hline T5 & Lambda cyhalothrin 5\% EC & $15 \mathrm{ml}$ a.i./ha & 89.23 \\
\hline T6 & Control (Untreated) & - & 43.72 \\
\hline
\end{tabular}

Table 7: Mean yield data of chilli to application of different insecticides against different insect pest of chilli crops during Rabi 2018-19

\begin{tabular}{|c|c|c|c|}
\hline S. No. & Name of treatment & Dose & Green Chilli Yield (q/ha) \\
\hline T1 & Emamectin Benzoate 3.5\% + lambda cyhalothrin 5\% WP & $11 \mathrm{~g}$ a.i./ha & 98.83 \\
\hline T2 & Emamectin Benzoate 3.5\% + lambda cyhalothrin 5\% WP & $16 \mathrm{~g}$ a.i./ha & 102.12 \\
\hline T3 & Emamectin Benzoate 3.5\% + lambda cyhalothrin 5\% WP & $21.25 \mathrm{~g}$ a.i./ha & 107.53 \\
\hline T4 & Emamectin Benzoate 5\% SG & $10 \mathrm{~g}$ a.i./ha & 86.00 \\
\hline T5 & Lambda cyhalothrin 5\% EC & $15 \mathrm{ml} \mathrm{a.i./ha}$ & 92.25 \\
\hline T6 & Control (Untreated) & - & 54.62 \\
\hline
\end{tabular}

Table 8: Pooled mean yield data of chilli to application of different insecticides against different insect pest of chilli crops during Rabi 2017-18 and 2018-19

\begin{tabular}{|c|c|c|c|}
\hline S. No. & Name of treatment & Dose & Green Chilli Yield (q/ha) \\
\hline T1 & Emamectin Benzoate 3.5\% + lambda cyhalothrin 5\% WP & $11 \mathrm{~g}$ a.i./ha & 97.31 \\
\hline T2 & Emamectin Benzoate 3.5\% + lambda cyhalothrin 5\% WP & $16 \mathrm{~g}$ a.i./ha & 100.57 \\
\hline T3 & Emamectin Benzoate 3.5\% + lambda cyhalothrin 5\% WP & $21.25 \mathrm{~g}$ a.i./ha & 106.02 \\
\hline T4 & Emamectin Benzoate 5\% SG & $10 \mathrm{~g}$ a.i./ha & 84.51 \\
\hline T5 & Lambda cyhalothrin 5\% EC & $15 \mathrm{ml}$ a.i./ha & 90.75 \\
\hline T6 & Control (Untreated) & - & 49.17 \\
\hline
\end{tabular}

\section{Results and Discussion}

The insecticide molecules Emamectin Benzoate 3.5\% + lambda cyhalothrin 5\% WP @ $11 \mathrm{~g}$ a.i./ha, Emamectin Benzoate 3.5\% + lambda cyhalothrin 5\% WP @ 16 g a.i./ha, Emamectin Benzoate 3.5\% + lambda cyhalothrin 5\% WP @ $21.25 \mathrm{~g}$ a.i./ha, Emamectin Benzoate 5\% SG @ $10 \mathrm{~g}$ a.i./ha, Lambda cyhalothrin 5\% EC @ $15 \mathrm{~g}$ a.i./ha and untreated control were evaluated for their efficacy manage the pest complex of chilli.
Bio efficacy of Emamectin Benzoate 3.5\% + lambda cyhalothrin 5\% WP against sucking pest on chilli Thrips

The non - significant difference was observed in different plots during the pretreatment observation. A day after application of insecticides, the minimum thrips population per plant observed in Emamectin Benzoate 3.5\% + lambda cyhalothrin 5\% WP @ $21.25 \mathrm{~g}$ a.i./ha (10.21 thrips/plant) followed by Emamectin Benzoate 3.5\% + lambda cyhalothrin 
5\% WP @ 16 g a.i./ha (13.48 thrips/plant), Emamectin Benzoate 3.5\% + lambda cyhalothrin 5\% WP @ $11 \mathrm{~g}$ a.i./ha (17.96 thrips/plant), Lambda cyhalothrin 5\% EC @ $15 \mathrm{~g}$ a.i./ha (20.76 thrips/plant) and in Emamectin Benzoate 5\% SG @ 10 g a.i./ha (21.61 thrips/plant). However, the maximum thrips population (30.62 thrips/plant) was noticed in untreated control. Sathyan et al. (2017) ${ }^{[18]}$ revealed that significantly lowest population of thrips was recorded in fipronil 5 SC @ 0.15\% (8.05 thrips/3 bud) and Imidacloprid 17.8 SL @ 0.02\% (9.0 thrips/3 bud) in chilli. Nagaraj et al. (2007) ${ }^{[14]}$ revealed that the mean thrips population recorded were minimum in thiomethaxam 25 WG @ $35 \mathrm{~g}$ a.i./ha (2.95 thrips/leaf).

\section{Mite}

Mite population was recorded before and after one, three, seventh, tenth and fifteenth days after spraying of insecticides. Pretreatment mite population was observed to be statistically non-significant. The observation recorded on one day after spraying of insecticides displayed that, all the insecticidal treatments were significantly superior over control in reducing the infestation of mite population. Among all the five insecticidal treatments, the foliar application of Emamectin Benzoate 3.5\% + lambda cyhalothrin 5\% WP @ $21.25 \mathrm{~g}$ a.i./ha had the lowest $2.04 \mathrm{mite} / \mathrm{plant}$. (T2) and (T1) i.e. Emamectin Benzoate $3.5 \%$ + lambda cyhalothrin 5\% WP @ $16 \mathrm{~g}$ a.i./ha, Emamectin Benzoate 3.5\% + lambda cyhalothrin 5\% WP @ $11 \mathrm{~g}$ a.i./ha was found statistically on at par with each other. Samanta et al. (2017) ${ }^{[17]}$ stated that the treatments, spiromesifen 24 SC @ $120 \mathrm{~g}$ a.i./ha were observed to be very much effective against yellow mite. Shahaji (2007) [19] stated that the abamectin $0.0009 \%$ was significantly superior in reducing mite population with 2.66 mites/plant.

\section{Aphid}

The application of Emamectin Benzoate 3.5\% + lambda cyhalothrin 5\% WP @ $21.25 \mathrm{~g}$ a.i./ha, (3.51 aphid/plant) recorded the least aphid population per plant, followed by (T2) Emamectin Benzoate 3.5\% + lambda cyhalothrin 5\% WP @ $16 \mathrm{~g}$ a.i./ha,(4.47 aphid/plant) and (T1) Emamectin Benzoate 3.5\% + lambda cyhalothrin 5\% WP @ $11 \mathrm{~g}$ a.i./ha,(5.11 aphid/plant). Ditya et al. (2010) ${ }^{[5]}$ stated that chlorfenapyr belongs to pyrrole group of insecticides and is used as a broad spectrum insecticide cum acaricide for the control of whiteflies, thrips, caterpillar, mites, leaf miners, aphids etc. Furthermore, effectiveness of imidacloprid was reported by various workers against aphid on chilli. Kumar et al. (2001) ${ }^{[12]}$ stated thatimidacloprid (70 g/ha) was the best treatment in controlling aphids (99.76\% reduction) against the major pest complex aphids, thrips, gram pod borer, tobacco caterpillar of chilli. Das (2013) ${ }^{[4]}$ concluded that imidacloprid had good knock down effect on aphids population in chilli. Sujay et al. (2015) [21] noted lesser effectiveness of imidacloprid against chilli pests. Viz. green peach aphid (Myzus persicae Sulzer, Aphis gosspyi Glover) and other sucking pests.

\section{Effect of insecticide on the natural enemies of insect pest on chilli \\ Coccinellid}

The observations on coccinellids were recorded after first spray revealed that, a non-significant difference among various treatments indicating that the predator was spread in all the treatments. The overall mean population of coccinellids after first spray, each spray indicated that untreated control (0.46 coccinellids/plant) and Emamectin Benzoate 3.5\% + lambda cyhalothrin 5\% WP @ $21.25 \mathrm{~g}$ a.i./ha, Emamectin Benzoate 3.5\% + lambda cyhalothrin 5\% WP @ $16 \mathrm{~g}$ a.i./ha and Emamectin Benzoate 3.5\% + lambda cyhalothrin 5\% WP @ 11 g a.i./ha $(0.42,0.41$ and 0.43 coccinellids/plant) recorded relatively higher population of coccinellids. Emamectin Benzoate 5\% SG @ $10 \mathrm{~g}$ a.i./ha and Lambda cyhalothrin 5\% EC @ $15 \mathrm{ml}$ a.i./ha has pared lower predator population compare to other treatments. The data pertaining to the impact of Emamectin Benzoate 3.5\% + lambda cyhalothrin 5\% WP on the occurrence of natural enemies are presented in Table 4. Shinde et al. (2007) ${ }^{[20]}$ reported that spinosad 45 SC @ $75 \mathrm{~g}$ a.i./ha was most safer insecticide to the predators on okra.

\section{Spiders}

Although Emamectin Benzoate 5\% SG @ $10 \mathrm{~g}$ a.i./ha $(0.63$ spider/plant) and lambda cyhalothrin 5\% EC @ $15 \mathrm{ml}$ a.i./ha (0.60 spider/plant) recorded lower population compared to other treatments in second sprays. In general, untreated control T6 (0.70 spider/plant), T3 (0.64 spider/plant), T2 (0.65 spider/plant) and T1 (0.67 spider/plant) treatments registered higher population as compared to other treatments.

\section{Fruit yield and economic assessment}

The data of two years mean total healthy green chilli fruit yield of all the treatment was significant higher over untreated control. At the end of the experiment, the marketable fruit yield (Table 8 ) of all the picking was added and transformed into quintals on hectare basis. Among all the treatments (T3), Emamectin Benzoate 3.5\% + lambda cyhalothrin 5\% WP @ $21.25 \mathrm{~g}$ a.i./ha. proved to be the best in producing highest marketable yield (106.02 q/ha.) followed by (T2) Emamectin Benzoate 3.5\% + lambda cyhalothrin 5\% WP @ $16 \mathrm{~g}$ a.i./ha (100.57 q/ha.) at par with (T1) Emamectin Benzoate 3.5\% + lambda cyhalothrin 5\% WP @ $11 \mathrm{~g}$ a.i./ha (97.31 q/ha) and single dose (T5) lambda cyhalothrin 5\% EC @ $15 \mathrm{ml}$ a.i./ ha (90.75 q/ha). Comparatively low yield was recorded in Emamectin Benzoate 5\% SG @ 10 g a.i./ ha (T4) (84.51 $\mathrm{q} / \mathrm{ha})$. The lowest fruit yield was recorded in control (49.17 q/ha). (T3) Emamectin Benzoate 3.5\% + lambda cyhalothrin 5\% WP @ $21.25 \mathrm{~g}$ a.i./ha was found significantly superior (at $5 \%$ ) over all other treatments in giving the highest yield. Patra et al. (2016) ${ }^{[16]}$ observed that the chlorantraniliprole was the best treatment in reducing the shoot infestation and gave highest marketable brinjal fruit yield (155.01 q/ha).

\section{Conclusion}

The new chemical insecticide Emamectin Benzoate 3.5\% + lambda cyhalothrin 5\% WP was evaluated for its bio-efficacy against sucking pest of chilli in the department of entomology, IGKV, Raipur during Rabi 2017-18 and 201819. During the bio-efficacy trial, the effect of all tested doses of Emamectin Benzoate 3.5\% + lambda cyhalothrin 5\% WP effectively control the population of sucking insect pest of chilli. It was also observed that Emamectin Benzoate 3.5\% + lambda cyhalothrin 5\% WP at all dosages levels tested for bio efficacy has non-significant lower population of natural enemies under field condition.

\section{References}

1. Anonymous. Indian Horticulture Database, Ministry of Agriculture, Government of India, 2017, 6.

2. Ahmed K, Mohamed MG, Murthy NSR. Yield losses due to various pests in hot pepper. Capsicum Newsletter. 1987; 6:83-84. 
3. Choudhary BR, Fageria S. A text book on production technology of vegetables (Kalyani publishers). 2009; 2:66-67.

4. Das G. Efficacy of imidacloprid, a nicotinoid group of insecticide against the infestation of chilli aphid, Myzus persicae (Hemiptera : Aphididae). Int. J. of Agri. Inno. and Res. 2013; 4(4):5.

5. Ditya P, Das SP, Sarkar PK, Bhattarchryya A. Degradation dynamics of chlorfenapyr residue in chilli, cabbage and soil. Bulletin of Environ. Contami. and toxicology. 2010; 84(5):602-605.

6. Gill HS. Improved technologies for chilli production. Indian Cocoa Arecanut and spices Journal. 1989; 12:118119.

7. Halder J, Kodandaram H. Emerging insect pest problems in vegetable crops and their management in India: An appraisal. Pest Management in Horticultural Ecosystems. 20(2):113-122.

8. Joia BS, Jaswinder K, Udean AS. Persistence of ethion residue on/in green chilli. Proceeding of National Symposium on Integrated Pest Management in Agriculture Crops, Bagalore, 2001, 174-175.

9. Kandasamy C, Mohanasundaram M, Karuppachamy P. Evaluation of insecticides for the control of thrips, Scirtothrips dorsalis Hood, in chillis (Capsicum annum L.). Madras Agriculture Journal. 1990; 77(3-4):169-172.

10. Kraft $\mathrm{KH}$, Brown $\mathrm{CH}$, Nabhan GP, Luedeling E, Ruiz JL, dEeckenbrugge GC et al. Multiple lines of evidence for theorigin of domesticated chili pepper, Capsicum annuum, in Mexico. PNAS. 2014; 111:6165-70.

11. Kumar NKK. Yield loss in chilli and sweet pepper due to Scirtothrips dorsalis (Hood). (Thysanoptera: Thripidae). Pest Management in Horticultural Ecosystems. 1995; 1(2):61-69.

12. Kumar KP, Reddy DJ, Narendranath VV. Bio-efficacy of selected insecticides against pest complex in chilli (Capsicum annuum) pesticide res. J. 2001; 13(1):36-41.

13. Mondal B, Mondal P. Eco-friendly pest management practices for leaf curl complex of chilli Capsicum annum (L.). Biopest, (supplementary). 2012; 4:115-118.

14. Nagaraj T, Sreenivas AG, Patil BV, Nagangoud A. Preliminary evaluation of some new molecules against thrips Scirtothrips dorsalis Hood and Polyphagotarsonemus latus Banks mites in chilli under irrigated ecosystem. Pest Management in Horticultural Ecosystems. 2007; 13(2):185-188.

15. Nelson SJ, Natarajan S. Economic threshold level of thrips in semi-dry chilli. South Indian Horticulture. 1994; 42(5):336-338.

16. Patra S, Thakur NSA, Firake DM. Evaluaion of Biopesticides and insecticides against brinjal shoot and fruit borer (leucinodes orbonalis Guenee). Int. J Bio-resource and Stress Management. 2016; 7(5):1032-1036.

17. Samanta A, Sen k, Basu I. Evaluation of insecticides and acaricides against yellow mite and thrips infesting chilli (Capsicum annum L.). Journal of Crop and Weed. 2017; 13(2):180-186.

18. Sathyan T, Dhanya MK, Preethy TT, Aswathy TS, Mueugan M. Relative efficacy of some newer molecules against thrips, Scirtothrips dorsalis Hood (Thysanoptera: Thripidae) on rose. Journal of Entomology and Zoology Studies. 2017; 5(3):703-706.

19. Shahaji SK. Seasonal incidence and bio-efficacy of newer insecticides against pest infesting chilli, Capsicum annum L. M.Sc. (Ag.) Thesis, Mahatma Phule Krishi Vidyapeeth, Rahuri, 2007, 39p.

20. Shinde BD, Sarkate MB, More SA, Sable YR. Evaluation of different pesticide for safetyness to predators on okra. Pestology. 2007; 31:25-28.

21. Sujay YH, Giraddi RS, Udikeri SS. Efficacy of new molecules and botanicals against chilli (Capsicum annuит L.) pests. Agricultural J. 2015; 102:348-352. 